\title{
Aufstiegskompetenz von Frauen
}

\author{
Monique Janneck • Sylvie Vincent-Höper
}

Auch im Jahre 2012 ist noch eine Aufteilung der Erwerbsarbeit nach Geschlecht festzustellen: Frauen sind sowohl in bestimmten Branchen und Berufen, insbesondere im technisch-naturwissenschaftlichen Bereich, als auch in Führungspositionen nach wie vor unterrepräsentiert. Insbesondere auf den höheren und höchsten Führungsebenen ist der Frauenanteil immer noch minimal.

Um in die oberen Etagen betrieblicher Hierarchien zu gelangen, ist neben Fachkompetenz spezifische Aufstiegskompetenz erforderlich: nämlich die Fähigkeit und Bereitschaft, sich Führungspositionen zu erschließen. Mit Aufstiegskompetenz ist somit die emotional-motivationale und kognitive Bereitschaft sowie auch die Befähigung zur beruflichen Weiterentwicklung und zur Erschließung von Führungspositionen gemeint. Sie wirkt als Metakompetenz und umfasst verschiedene interagierende Motivations- und Kompetenzfacetten.

Diesem Themenkomplex widmete sich das Forschungsprojekt „Aufstiegskompetenz von Frauen“, einem Verbundprojekt der Universitäten Hamburg und Leipzig, das von Frühjahr 2009 bis Ende 2011 vom BMBF im Rahmen der Bekanntmachung „Frauen an die Spitze“ gefördert und vom ESF kofinanziert wurde. In diesem Forschungsvorhaben wurden förderliche und hinderliche Faktoren von Aufstiegskompetenz untersucht und Gestaltungsvorschläge entwickelt, durch die der Anteil von Frauen an Führungspositionen gefördert werden kann. Besondere Aufmerksamkeit galt dabei Branchen und Berufen mit geringem Frauenanteil, wie insbesondere den schon erwähnten technisch-naturwissenschaftlichen Bereichen (siehe dazu die Homepage des Verbundprojektes: http://www. aufstiegskompetenz.de).

Dabei wurde der Blickwinkel nicht nur auf die Rolle der Frauen selber, ihre Motivation und Kompetenzen gelegt, sondern auch auf die situativen Bedingungen, wie insbe-

\author{
Online publiziert: 19.06 .2012 \\ (C) VS Verlag für Sozialwissenschaften 2012 \\ Prof. Dr. M. Janneck $(\bowtie)$ \\ Fachbereich Elektrotechnik und Informatik, Fachhochschule Lübeck, \\ Mönkhofer Weg 239, 23562 Lübeck, Deutschland \\ E-Mail: monique.janneck@fh-luebeck.de \\ Dr. S. Vincent-Höper \\ Arbeitsbereich Arbeits- und Organisationspsychologie, \\ Fachbereich Psychologie, Universität Hamburg, \\ Von-Melle-Park 11, 20146 Hamburg, Deutschland \\ E-Mail: sylvie.vincent@uni-hamburg.de
}


sondere der Einfluss der Arbeitsbedingungen und Merkmale der Arbeitsaufgabe auf die Aufstiegskompetenz.

Die fünf Beiträge im Thementeil „Aufstiegskompetenz“ beleuchten verschiedene Facetten und Ergebnisse des Forschungsvorhabens:

Der erste Beitrag von Sabine Korek und Thomas Rigotti (Aufstiegsförderliche Führung - gut für den Aufstieg, schlecht für die Gesundheit?) thematisiert die Rolle der Führungskräfte für die Entwicklung von Aufstiegskompetenz bei ihren Mitarbeiterinnen und Mitarbeitern. Aufstiegsförderliche Führung zeichnet sich demnach durch vier Dimensionen aus - Delegation, Feedback, Vertrauen und Förderung - die vorgestellt und deren Zusammenhang mit dem Berufserfolg anhand empirischer Untersuchungen aufgezeigt wird. Die AutorInnen greifen in ihrem Beitrag jedoch auch die Frage auf, inwiefern ein solches stark auf die Karriereentwicklung ausgerichtetes Führungsverhalten möglicherweise negative Konsequenzen für die Gesundheit der Mitarbeiterinnen und Mitarbeiter mit sich bringt.

Mikropolitik, also informelle Formen und Strategien der Einflussnahme, um eigene Interessen durchzusetzen, spielt eine große Rolle für Strukturen und Prozesse in Unternehmen - auch für den Aufstieg von Frauen in Führungspositionen? Dieser Frage gehen Doris Cornils, Anna Mucha und Daniela Rastetter (Zur Bedeutung von mikropolitischer Kompetenz für den Aufstieg von Frauen in Führungspositionen - am Beispiel der Handlungsfelder Unternehmenskultur und Selbstdarstellung) in ihrem Beitrag nach und beantworten sie mit einem klaren Ja. Zu diesem Ergebnis kommen die Autorinnen anhand einer umfangreichen Interventionsstudie, in deren Rahmen 30 weibliche Nachwuchsführungskräfte ein Mikropolitik-Coaching erhielten. Der Beitrag stellt das von den Autorinnen entwickelte Mikropolitische Kompetenzmodell (MKM) vor und erläutert die Bedeutsamkeit von mikropolitischer Kompetenz für den Aufstieg an Beispiel der Handlungsfelder Unternehmenskultur und Selbstdarstellung.

Der Beitrag von Angelika Wagner, Telse Iwers-Stelljes, Judith Oerding und Inken Paulsen thematisiert Mentale Blockaden der Aufstiegskompetenz von Frauen. Der Beitrag führt in das Konstrukt mentaler Blockaden sowie die Theorie mentaler Introferenz ein, um dann konkret auf die Rolle mentaler Blockaden für die Entwicklung, Entfaltung und Anwendung von Aufstiegskompetenz einzugehen. Die Autorinnen stellen einen Fragebogen zur Erhebung mentaler Blockaden in Schlüsselsituationen des beruflichen Aufstiegs sowie eine Interventionsstudie vor, in deren Rahmen ein Introvisions-Coaching zur Auflösung mentaler Blockaden mit insgesamt 52 weiblichen Nachwuchsführungskräften durchgeführt wurde. Die Ergebnisse zeigen, dass die Teilnahme an dem Coaching zu einer signifikanten Verringerung von mentalen Blockaden sowie von Stress und Belastung führte.

Auch der nachfolgende Beitrag von Janina Pflugradt und Monique Janneck befasst sich mit mentalen Blockaden von Frauen (,Ein bisschen wie ein Außerirdischer “: Subjektive Imperative und mentale Blockaden von Frauen in technisch-naturwissenschaftlichen Berufsfeldern). Konkret geht der Beitrag der Frage nach, inwiefern mentale Blockaden existieren, die das berufliche Vorankommen von Frauen speziell in technischen Berufen beeinträchtigen könnten. Hierzu wurden qualitative Interviews mit weiblichen Führungskräften bzw. Nachwuchskräften anhand des imperativtheoretischen Textanalyseverfahrens sekundäranalytisch untersucht. Zahlreiche so genannte subjektive Imperative 
konnten identifiziert werden, aus denen bei Nichterfüllung bzw. Kollisionen mit anderen Imperativen mentale Blockaden entstehen können. Praktische Implikationen für die Förderung der Teilhabe von Frauen an „Männerberufen“ werden diskutiert.

Der abschließende Beitrag von Monique Janneck, Sylvie Vincent-Höper und Ina Othersen (Entwicklung und Validierung eines Fragebogens zum Technikbezogenen Selbstkonzept (TSK): Eine gendersensitive Studie) thematisiert ebenfalls die Unterrepräsentanz von Frauen in technisch-ingenieurswissenschaftlichen Berufsfeldern. Mit dem technikbezogenen Selbstkonzept wird ein Konstrukt vorgestellt, das dazu beitragen kann, diese Unterrepräsentanz zu erklären und zu analysieren. Hierzu wurde mit dem Fragebogen zum Technikbezogenen Selbstkonzept (TSK) ein geschlechtersensitives Instrument entwickelt und validiert, das u. a. zur Identifikation von Schwächen bzw. Defiziten hinsichtlich des TSK eingesetzt werden kann, die sich negativ auf den Berufserfolg auswirken könnten, um entsprechende Handlungsempfehlungen abzuleiten. 